'Departamento de Ciencias Sociales, Facultad de Educación y Humanidades, Universidad del Bío-Bío, Chillán, Chile. apsicólogo, Magíster en Psicología Social.

Recibido el 9 de diciembre de 2014, aceptado el 30 de junio de 2015.

Conflictos de intereses: nada que declarar.

Correspondencia a: Rodolfo Mendoza Llanos Andrés Bello S/N, Escuela de Psicología. Chillán, Chile. Teléfono: 0422463238 rmendoza@ubiobio.cl

\section{Insatisfacción laboral como predictor del ausentismo en un hospital público}

\author{
RODOLFO MENDOZA LLANOS ${ }^{a}$
}

\section{Job satisfaction and organizational culture as predictors of absenteeism}

Background: Absenteeism caused by sick leaves generates an important economic burden. Aim: To determine if job satisfaction, psychological climate, hierarchic level and age are predictors of absenteeism caused by sick leaves. Material and Methods: FOCUS-93 survey that measures organizational culture and the satisfaction scale of Warr, Cook and Wall were distributed to 1387 workers of a hospital and answered by 874. Absenteeism data was obtained from the justified absenteeism registry of the Chilean Health Services. Results: Absenteeism is influenced by job satisfaction and organizational culture. Age has no influence. Hierarchic level has a negative influence on absenteeism among men and a negligible effect among women. Conclusions: Those workers that are not satisfied with their work have higher rates of absenteeism.

(Rev Med Chile 2015; 143: 1028-1033)

Key words: Absenteeism; Hospital; Job satisfaction; Organizational culture.
1 ausentismo laboral se entiende como la falta de un trabajador en su lugar de trabajo por razones ajenas al desempeño del mismo ${ }^{1}$. En Chile, el ausentismo laboral por causa médica es la primera de las tres causas que generan mayor gasto público en salud ${ }^{2}$. Dado que los hospitales de alta complejidad funcionan $24 \mathrm{~h}$, el ausentismo por licencias médicas por enfermedad tiene un costo social asociado que se produce cuando se debe reemplazar a la persona ausente de manera inmediata, ya sea contratando personal o readecuando los turnos. Esta última alternativa es la más usual y significa que los trabajadores deban asumir una sobrecarga, ya sea realizando turnos extras o supervisando el personal menos experto, lo que va en directo detrimento de su bienestar físico, tiempo para las actividades extralaborales y calidad de vida ${ }^{3,4}$, lo que afecta su satisfacción laboral, al ser el trabajo una fuente permanente de cambio. La gestión del ausentismo en hospitales y su relación con la satisfacción laboral ha suscitado varios estudios en las últimas décadas ${ }^{1,5-12}$.

$\mathrm{Al}$ revisar diversos predictores de ausentismo laboral de acuerdo a los efectos que tienen en modelos predictivos, se pueden clasificar en tres grandes grupos: los culturales, que afectan a la población general de un país o región o son referidos a la cultura de una empresa u organización ${ }^{13}$; los situacionales, referidos a agentes externos al sujeto que tienen origen en las prácticas, roles y procedimientos propios de una organización, un lugar específico en la organización o un rol en la sociedad y los individuales, que consideran aquellas variables que son propias del individuo y que escapan de los límites de una organización.

Dentro de los predictores individuales los que tienen más apoyo empírico son el género ${ }^{14-18}$, y la edad ${ }^{13-17,19}$. Otras variables que han tratado de vincularse al ausentismo sin un apoyo empírico concreto son la salud del trabajador ${ }^{9,19,20}$, número de hijos ${ }^{9,13}$ y aquellas que no han encontrado resultados estadísticamente significativos son el estado civil $^{9,13}$ y el compromiso organizacional ${ }^{19,20}$.

Dentro de los predictores situacionales de ausentismo con mayor apoyo están el nivel jerárquico $^{9,13,14,17}$, la remuneración ${ }^{9,20}$ y el clima 
psicológico ${ }^{16,21,22}$. Otros predictores situacionales estudiados sin apoyo claro son permisividad organizacional $l^{9,20}$, conflicto de $\mathrm{rol}^{9}$ y estabilidad en el trabajo ${ }^{9,13}$.

La satisfacción laboral es una variable mediadora entre los aspectos individuales y los situacionales, en tanto, tiene ámbito intrínsecos y extrínsecos, respectivamente ${ }^{23}$. Su relación con el ausentismo se establece al plantearse que las ausencias de corto plazo ( 1 o 2 días) son más propensas a estar a discreción del trabajador, o con la voluntad de ausentarse, pudiendo abusar de ellas ${ }^{24}$ lo que se reflejaría en la frecuencia de ausentismo, lo que tiene apoyo en modelos que identifican las licencias médicas fraudulentas, como aquellas que tienden a ser más breves, recurrentes y de patologías de difícil comprobación ${ }^{25}$.

El ausentismo más alto en Chile se da en el sector hospitalario ${ }^{1}$, en un contexto laboral que se caracteriza por el cansancio asociado a turnos de trabajo, limitada posibilidad de capacitación y escasez de personal por ausentismos que conmina la realización de trabajo extra, impactando negativamente los niveles de satisfacción laboral ${ }^{26}$ y afectando a su vez, la calidad de la atención de pacientes $^{26,27}$.

Dados los antecedentes anteriores el propósito de este estudio fue evaluar el ajuste de un modelo predictivo de ausentismo por enfermedades de morbilidad común basado en la interacción de las siguientes variables: satisfacción laboral, variables situacionales (nivel jerárquico y clima psicológico) e individuales (edad y género). Para evaluar el impacto del género, se ponen a prueba tres modelos: uno para hombres, otro para mujeres y uno mixto, los que permiten determinar la dirección de las relaciones entre satisfacción laboral y el ausentismo. Las relaciones propuestas como hipótesis se pueden observar en la Figura 1.

\section{Material y Método}

Se distribuyeron 1.387 encuestas, entre trabajadores de un hospital de alta complejidad de las cuales se recuperaron solo 874 completas de 30 unidades distintas, constituyendo la muestra del presente estudio, el que es de carácter transversal, descriptivo, correlacional y no experimental.

La distribución por sexo es 71,2\% mujeres. De acuerdo a las edades el 29,2\% tiene edades entre 20 y 29 años, el 25,3\% tiene entre 30 y 39 años, el $25,3 \%$ tiene entre 40 y 49 años, el 18,2\% tienen entre 50 y 59 años y solo el 2,1\% tiene más de 60 años. Respecto del estamento el 13,6\% fueron auxiliares; $9,6 \%$ personal administrativo; $42,9 \%$ personal técnico; $25,7 \%$ profesionales no médicos y $8,1 \%$ médicos.

Los instrumentos utilizados fueron: FOCUS-93, instrumento que mide cuatro dimen-

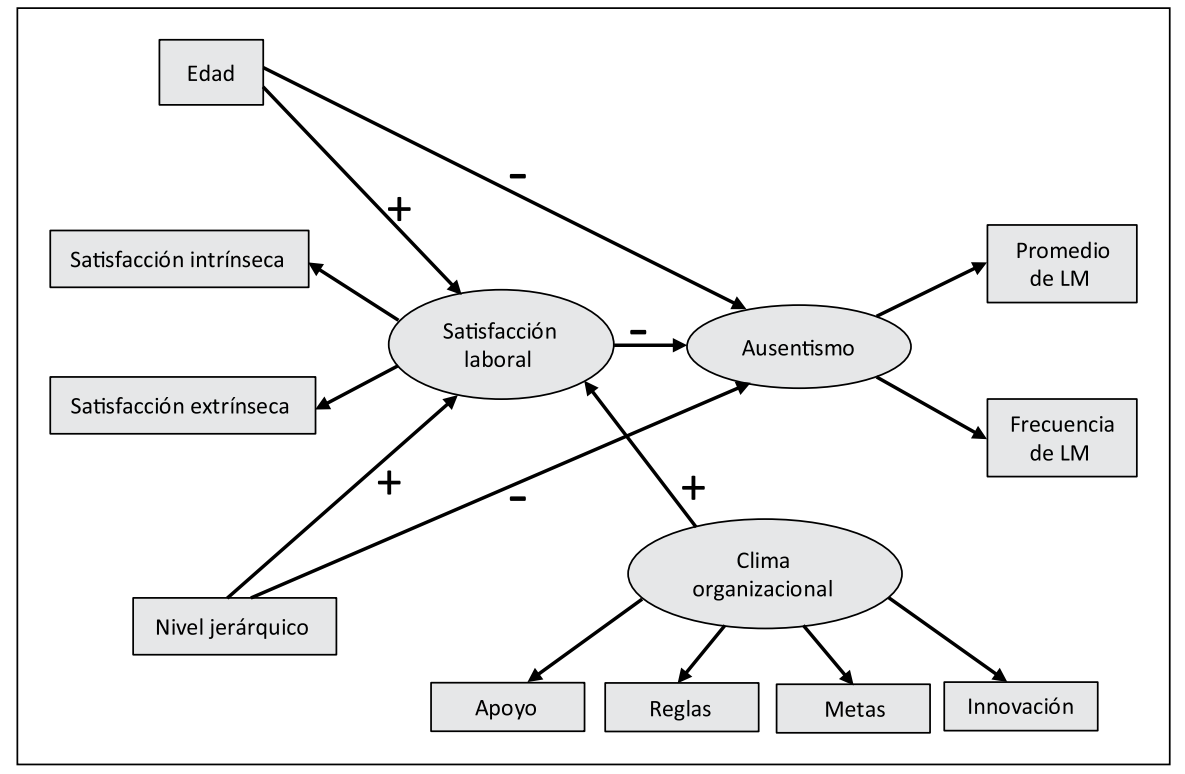

Figura 1. Especificación del modelo hipotetizado. 
siones del clima psicológico: Metas, Innovación, Reglas y Apoyo, y la Escala General de Satisfacción de Warr, Cook y Wall (1979) basado en la teoría de Herzberg de dos factores que indica satisfacción intrínseca y extrínseca ${ }^{23}$, cuya fiabilidad estimada mediante alfa de Cronbach para muestras chilenas va desde 0,85 a 0,88 . Este instrumento enfatiza la actitud personal, por sobre la percepción del entorno socio-laboral, lo que lo diferencia de clima psicológico $^{28}$.

Para ausentismo se utilizaron valores poblacionales de la institución: frecuencia y media de días de acuerdo a estamento y unidad de trabajo ocurridos en el mes inmediatamente posterior a la aplicación del cuestionario para la población definida, obtenidos a través del informe de ausentismo justificado detallado del Sistema de Información de Recursos Humanos (SIRH) de los Servicios de Salud de Chile. No se utilizaron los autoreportes de ausentismo puesto que éstos tienen como limitación el que las personas tienden a desestimar su ausentismo ${ }^{18}$.

Dado las estructuras organizacionales en salud tienden a entregar una remuneración mayor a quien tiene un mayor nivel jerárquico, se asume que el nivel jerárquico incluye las remuneraciones, y el nivel educacional (logrado por la cantidad de semestres estudiados) define el nivel jerárquico, se asume este último como años de estudio formal.

Los datos fueron procesados $y$ analizados utilizando el programa AMOS 18.0 para la modelización a través de ecuaciones estructurales ${ }^{29}$.

\section{Resultados}

De acuerdo a lo observado en la Tabla 1, los modelos hipotetizados presentan ajustes globales adecuados ${ }^{29}$, explicándose en cada uno de ellos más de $94 \%$ de la varianza conjunta.

Los resultados, que se observan en la Tabla 2, apoyan una relación positiva entre edad y clima psicológico con satisfacción laboral, y una relación negativa entre satisfacción laboral y ausentismo para los tres modelos de género. Las relaciones negativas hipotetizadas para edad y nivel jerárquico con ausentismo son apoyadas parcialmente solo en el modelo que considera hombres y el mixto. Finalmente, la relación positiva hipotetizada entre el nivel jerárquico y la satisfacción laboral solo se observa en el modelo de los hombres.

De acuerdo a los resultados obtenidos para todos los modelos evaluados (Tabla 3), la satisfacción laboral es la variable que tiene mayor efecto sobre el ausentismo.

Al comparar los efectos totales, el modelo para hombres es menor que el obtenido en el modelo para mujeres, el que es similar al efecto del modelo mixto. El efecto total del clima psicológico en el modelo para hombres es menor al del modelo para

Tabla 1. Índices de ajuste para los modelos según género

\begin{tabular}{|c|c|c|c|c|}
\hline Índices & Valor óptimo & $\begin{array}{l}\text { Modelo hombres } \\
\qquad(n=252)\end{array}$ & $\begin{array}{l}\text { Modelo mujeres } \\
\quad(n=622)\end{array}$ & $\begin{array}{l}\text { Modelo mixto } \\
\qquad(n=847)\end{array}$ \\
\hline \multicolumn{5}{|c|}{ Medidas de ajuste global } \\
\hline$\chi^{2}$ (g.l.); & $p<0,05$ & $70,831(32)$ & $11,925(31)$ & $153,866(31)$ \\
\hline $\mathrm{p}$ & & $p=0,001$ & $p=0,001$ & $p=0,001$ \\
\hline$\chi^{2} / g .1$. & Cercanos a 2 & 2,213 & 3,804 & 4,963 \\
\hline NCP & $p<0,05$ & 38,831 & 86,925 & 122,866 \\
\hline GFI & $>0,9$ & 0,947 & 0,963 & 0,965 \\
\hline RMSEA & $0,05-0,08$ & 0,070 & 0,067 & 0,067 \\
\hline \multicolumn{5}{|c|}{ Medidas de ajuste incremental } \\
\hline AGFI & $>0,9$ & 0,909 & 0,934 & 0,938 \\
\hline TLI & $>0,9$ & 0,935 & 0,924 & 0,927 \\
\hline $\mathrm{CFI}$ & Cercanos a 1 & 0,954 & 0,948 & 0,950 \\
\hline \multicolumn{5}{|c|}{ Medidas de ajuste de parsimonia } \\
\hline PNFI & 0,06-0,09 & 0,654 & 0,641 & 0,646 \\
\hline AIC & Menor valor & 116,831 & 165,925 & 201,866 \\
\hline PGFI & Valor alto & 0,551 & 0,543 & 0,544 \\
\hline
\end{tabular}


Tabla 2. Estimadores de ruta del modelo

\begin{tabular}{|c|c|c|c|}
\hline Ruta del modelo estructural & Hombres & Mujeres & Mixto \\
\hline Edad $\rightarrow$ Satisfacción laboral & 0,016 & 0,049 & 0,041 \\
\hline Nivel Jerárquico $\rightarrow$ Satisfacción laboral & 0,015 & $-0,028$ & $-0,008$ \\
\hline Clima psicológico $\rightarrow$ Satisfacción laboral & 0,677 & 0,623 & 0,637 \\
\hline Satisfacción Laboral $\rightarrow$ Ausentismo & $-0,108$ & $-0,274$ & $-0,270$ \\
\hline Edad $\rightarrow$ Ausentismo & $-0,023$ & 0,046 & $-0,040$ \\
\hline Nivel Jerárquico $\rightarrow$ Ausentismo & $-0,382$ & 0,067 & $-0,159$ \\
\hline Ausentismo $\rightarrow$ Promedio de licencias médicas & 0,993 & 0,372 & 0,521 \\
\hline Ausentismo $\rightarrow$ Frecuencia de licencias médicas & 0,175 & 0,633 & 0,391 \\
\hline Satisfacción Laboral $\rightarrow$ Satisfacción intrínseca & 0,928 & 0,857 & 0,877 \\
\hline Satisfacción Laboral $\rightarrow$ Satisfacción extrínseca & 0,926 & 0,968 & 0,956 \\
\hline Clima psicológico $\rightarrow$ Metas & 0,879 & 0,844 & 0,854 \\
\hline Clima psicológico $\rightarrow$ Reglas & 0,573 & 0,464 & 0,501 \\
\hline Clima psicológico $\rightarrow$ Apoyo & 0,651 & 0,622 & 0,630 \\
\hline Clima psicológico $\rightarrow$ Innovación & 0,708 & 0,669 & 0,683 \\
\hline
\end{tabular}

Todas las diferencias son significativas, con $\mathrm{p}<0,05$.

Tabla 3. Efectos según modelo de género de las variables edad, nivel jerárquico, satisfacción laboral y clima psicológico sobre el ausentismo por licencias médicas curativas

\begin{tabular}{|c|c|c|c|c|c|c|c|c|c|}
\hline & \multicolumn{3}{|c|}{$\begin{array}{c}\text { Efecto directo sobre } \\
\text { ausentismo }\end{array}$} & \multicolumn{3}{|c|}{$\begin{array}{c}\text { Efecto indirecto sobre } \\
\text { ausentismo }\end{array}$} & \multicolumn{3}{|c|}{$\begin{array}{c}\text { Efectos totales sobre } \\
\text { ausentismo }\end{array}$} \\
\hline & Hombres & Mujeres & Mixto & Hombres & Mujeres & Mixto & Hombres & Mujeres & Mixto \\
\hline Edad & $-0,023$ & 0,046 & $-0,040$ & $-0,002$ & $-0,013$ & $-0,011$ & $-0,025$ & 0,033 & $-0,051$ \\
\hline $\begin{array}{l}\text { Nivel } \\
\text { jerárquico }\end{array}$ & $-0,382$ & 0,067 & $-0,159$ & $-0,002$ & 0,008 & 0,002 & $-0,384$ & 0,074 & $-0,157$ \\
\hline $\begin{array}{l}\text { Satisfacción } \\
\text { laboral }\end{array}$ & $-0,108$ & $-0,274$ & $-0,270$ & --- & --- & --- & $-0,108$ & $-0,274$ & $-0,270$ \\
\hline $\begin{array}{l}\text { Clima } \\
\text { psicológico }\end{array}$ & --- & --- & --- & $-0,073$ & $-0,171$ & $-0,172$ & $-0,073$ & $-0,171$ & $-0,172$ \\
\hline
\end{tabular}

Todas las diferencias son significativas, con $p<0,05$.

mujeres y modelo mixto. El efecto total del nivel jerárquico en el modelo para hombres es más del doble que el modelo mixto, pero en un sentido contrario al resultado obtenido en el modelo para mujeres. La variable edad en los tres modelos tiene un impacto menor respecto del ausentismo.

\section{Discusión}

Se puede establecer que la edad no aparece como un predictor de ausentismo estable según género, pues las relaciones son distintas para hombres y mujeres, pero coincide con lo reportado por estudios anteriores ${ }^{16,17}$, aun así, solo explica un porcentaje menor del ausentismo, lo que coincide con planteamientos anteriores ${ }^{20}$ de que en ninguno de los casos su inclusión en un modelo aumenta de manera significativa la varianza explicada.

Respecto del nivel jerárquico y su impacto sobre el ausentismo se mantiene la relación planteada hipotéticamente en el modelo para hombres y mixto. Esta relación entregaría un respaldo a la idea de que el ausentismo puede ser funcional 
para disminuir el estrés en los niveles más bajos que presentan menor autonomía. En el modelo de las mujeres se da una relación positiva, pero que explica un porcentaje menor del ausentismo, lo que coincide varios estudios ${ }^{30}$ que plantean que las mujeres tienen mayor probabilidad de ausentarse independiente de las profesiones que tengan.

La satisfacción laboral en este estudio tiene un efecto superior al reportado en otros estudios ${ }^{20}$, lo que puede estar relacionado con la sugerencia que realizan de utilizar muestras grandes para evaluar esta relación, como es el caso de este estudio.

El clima psicológico puede implicar que los resultados varían orientándose a un aspecto más situacional del ausentismo, pues en todos los modelos tiene un gran impacto sobre la satisfacción laboral, pero los efectos sobre el ausentismo varían en su poder predictivo, siendo más débiles en los hombres que en las mujeres.

Las diferencias que se observan respecto del nivel jerárquico y el impacto que general la satisfacción laboral y el clima psicológico, orientan a pensar que los hombres estarían más determinados a permanecer en su trabajo por su posición por sobre las otras variables. En el caso de las mujeres su ausentismo estaría mayormente relacionado con su nivel de insatisfacción y del clima psicológico percibido, independiente del nivel jerárquico que posean dentro de la organización. Al estar más relacionado con el nivel de insatisfacción y relacionado con mayor frecuencia de ausentismo, el modelo apoya la idea de que las frecuencias de ausentismo capturan mejor la intención de ausentarse $\mathrm{e}^{24}$. Por otro lado, la duración de la licencia sería indicativo de mala salud e incluso relación con burnout ${ }^{30}$, lo que se puede observar en caso del modelo para hombres.

Uno de los aportes que presenta este estudio es que incorpora a todos los estamentos posibles en un hospital, permite evaluar la dinámica del ausentismo para todos ellos, lo que es un avance respecto de la mayoría de los estudios centrados en enfermeros y enfermeras realizados previamente ${ }^{30}$ y que continúan realizándose ${ }^{31}$.

En términos generales, una de las limitaciones que presentan los modelos para hombres y mujeres, es que en la práctica cotidiana hombres y mujeres trabajan en conjunto, por lo que si bien se pueden observar diferencias en la forma de describir y explicar el fenómeno del ausentismo por licencias médicas curativas, se debería realizar modelos longitudinales que permitan incorporar la auto correlación del ausentismo del lugar de trabajo con el ausentismo de los trabajadores, puesto que algunas "cubiertas de turno" que se puedan realizar por otro trabajador podrían traducirse en ausencia secundaria indirecta ${ }^{30}$.

Al ser el nivel jerárquico un indicador que tiene un poder predictivo importante se transforma en un buen punto de trabajo al momento de querer discriminar entre los grupos de trabajadores que se deben intervenir en términos de ausentismo laboral, pero cualquier actividad que busque controlar el ausentismo por licencia médica curativa podría transformarse en una mayor ausencia al disminuir el compromiso del empleado ${ }^{30}$, al no observarse por parte de éste una real intención de preocuparse por su bienestar y su satisfacción laboral, lo que podría transformarse en un nuevo motivo para que continúen "tirando licencias" médicas para realizar actividades personales ${ }^{17} \mathrm{O}$ evadir el trabajo como una medida de compensación hacia lo que pudiesen considerar un trato injusto de parte de la organización a través del comportamiento directivo ${ }^{30}$.

\section{Referencias}

1. Mesa F, Kaempffer AM. 30 años de estudio sobre ausentismo laboral en Chile: una perspectiva por tipos de empresas. Rev Med Chile 2004; 132: 1100-8.

2. Dirección de Presupuestos del Ministerio de Hacienda. Estadísticas de Recursos Humanos del Sector Público 2003-2012. Ministerio de Hacienda de Chile, Dirección de Presupuestos. Santiago 2013: I.S.B.N: 978-956-812367-3.

3. Jiménez A, Moyano E. Factores laborales de equilibrio entre trabajo y familia: medios para mejorar la calidad de vida. Universum 2008; 23 (1): 116-33.

4. Baez X, Galdámez C. Conflicto de rol familia-trabajo desde la perspectiva de los tipos de jornadas de trabajo. Revista de Psicología de la Universidad de Chile 2005; 14 (1): 113-23.

5. Albion M, Fogarty G, Machin M, Patrick J. Predicting absenteeism and turnover intentions in the health professions. Australian Health Review 2008; 32 (2): 271-81.

6. Becker S, Oliveira ML. Estudio sobre ausentismo en profesionales de enfermería del centro psiquiátrico de Manaus, Brasil. Rev Latino-Am Enfermagem 2008; 16 (1): 109-14.

7. García-Prado A, Chawla M. The impact of hospital ma- 
nagement reforms on absenteeism in Costa Rica. Health Policy Plan 2006; 21: 91-100.

8. van Dierendonck D, Le Blanc P, van Breukelen W. Supervisory behavior, reciprocity and subordinate absenteeism. Leadership \& Organization Developement Journal 2002; 23 (2): 84-92.

9. Goldberg C, Waldman D. Modeling employee absenteeism: testing alternative measures and mediated effect based on job satisfaction. Journal of Organizational Behavior 2000; 21: 665-76.

10. Silva D, Marziale MH. Absenteísmo de trabalhadores de enfermagem em um hospital universitário. Rev Latino-Am Enfermagem 2000; 8 (5): 44-51.

11. Gauci R, Norman I. Testing a model of absence and intent to stay in employement: a study of registred nurses in Malta. Int J Nurs Stud 1997; 34 (5): 375-84.

12. Hackett R, Bycio P. An evaluation of employee absenteeism as a coping mechanism among hospital nurses. Journal of Occupational and Organizational Psychology 1996; 69: 327-38.

13. Cohen-Mansfield J, Rosenthal A. Absenteeism of nursing staff in a nursing home. Int J Nurs Stud 1989; 26 (2): 187-94.

14. Praveen Parboteeah K, Addae H, Cullen J. National culture and absenteeism: a empirical test. International Journal of Organitational Analysis 2005; 13 (4): 343-61.

15. Hackett R, Guion R. A reevaluation of the absenteeism-Job satisfaction relationship. Organizational Behavior and Human Decision Process 1985; 35: 34081.

16. González-Romá V, Väänänem A, Ripoll P, Caballer A, Peiró JM, Kivimäki M. Psychological climate, sickness absence and gender. Psicothema 2005; 17 (1): 169-74.

17. Wegge J, Schmidt KH, Parkes C, van Dick R. 'Taking a sickie': Job satisfaction and job involvement as interactive predictors of absenteeism in a public organization. Journal of Occupational and Organizational Psychology 2007; 80: 77-89.

18. Hackett R. Work attitudes and employee absenteeism: A synthesis of literature. Journal of Occupational Psychology 1989; 62: 235-48.

19. Gellatly I. Individual and group determinants of employee absenteeism: test of a causal model. Journal of Organizational Behavior 1995; 16: 469-85.

20. Brooke P, Price J. The determinants of employee absen- teeism: An empirical test of a causal model. Journal of Occupational Psychology 1989; 62: 1-19.

21. Chiang M, Salazar M, Núñez A. Clima organizacional y satisfacción laboral en un establecimiento de salud pública: Hospital tipo 1. Theoria 2007; 16 (2): 61-76.

22. Medina F, Munduate L, Martínez I, Dorado M, Mañas M. Efectos positivos de la activación del conflicto de tarea sobre el clima de los equipos de trabajo. Revista de Psicología Social 2004; 19 (1): 3-15.

23. Alonso P. Diferencias en la percepción de la satisfacción laboral en una muestra de personal de administración. Boletín de Psicología 2006; 88: 49-64.

24. Scott K, Taylor GS. An examination of conflicting findings on the relationship between job satisfaction and absenteeism: a meta-analysis. Academy of Management Journal 1985; 28 (3): 599-612.

25. Mesa F, Raineri A, Maturana S, Kaempffer AM. Fraudes a los sistemas de salud en Chile: un modelo para su detección. Revista Panamericana de Salud Pública 2009; 25 (1): 56-61.

26. Fernández B, Paravic T. Nivel de satisfacción laboral en enfermeras de hospitales públicos y privados de la provincia de Concepción, Chile. Ciencia y Enfermería 2003; 9 (2): 57-66.

27. Parra S, Paravic T. Satisfacción laboral en enfermeras/ os que trabajan en el Sistema de Atención Médica de Urgencia (SAMU). Ciencia y Enfermería 2002; 8 (2): 37-48.

28. Cuadra A, Veloso C. Liderazgo, Clima y Satisfacción Laboral en las Organizaciones. Universum 2007; 22 (2): 40-56.

29. Schermelleh-Engel K, Moosbrugger H, Müller H. Evaluating the Fit of Structural Equation Models: Tests of Significance and Descriptive Goodness-of-Fit Measures. Methods of Psychological Research Online 2003; 8 (2): 23-74.

30. Belita A, Mbindyo P, English M. Absenteeism amongst health workers -developing a typology to support empiric work in low- income countries and characterizing reported associations. Human Resources for Health 2013; 11 (1): 34-44.

31. Trindade L, Gris C, Ostrovski V, Adamy E, Ferraz L, Amestoy S, et al. Absentismo en el equipo de enfermería en el ambiente hospitalario. Enfermería Global 2014; (36): 138-46. 